\title{
Track-based muon system alignment of the CMS detector
}

\author{
Hyunyong Kim ${ }^{\dagger}$ ** \\ Texas A\&M University, \\ 578 University Drive, College Station, TX 77843, USA \\ E-mail: hyunyong .kim@cern.ch
}

The alignment of the CMS muon detector is critical to maintain accurate position determination of muon hits, thereby affecting momentum resolution and the sensitivity of physics analyses involving muons in the final state. Muon track data from both the muon system and the inner tracker is used to perform a multidimensional fit on the misalignment degrees of freedom. The performance of this track-based alignment algorithm is validated by using muons in $\mathrm{Z}$ boson decays and evaluating the alignment's accuracy in reconstructing the mass peak. Chamber alignment accuracies on the order of $100 \mu \mathrm{m}$ are achieved and alignment performance is presented using Run 2 data.

40th International Conference on High Energy physics - ICHEP2020

July 28 - August 6, 2020

Prague, Czech Republic (virtual meeting)

\footnotetext{
$\dagger$ On behalf of the CMS Collaboration

*Speaker
} 


\section{Introduction}

The CMS muon system is designed to be highly redundant to reconstruct muons, measure muon momenta and charge, and trigger the CMS readout system upon muon detection over a broad kinematic range in challenging conditions including multiple particle interactions per collision. The alignment of chambers in the muon detector system is one of the important operational tasks to ensure stable trigger performance during data taking and excellent muon reconstructions in a wide variety of physics analyses.

\section{CMS Muon System}

The CMS muon system consists of three types of gaseous ionization chambers: drift tube chambers (DTs), cathode strip chambers (CSCs), and resistive plate chambers (RPCs). The DTs and CSCs measure an accurate position of the bending plane coordinate (measured as $R-\phi$ in the CSCs, where $\phi$ is the global azimuthal angle in cylindrical coordinates). The RPCs are primarily designed to provide timing information for the muon trigger, so the DTs and CSCs are considered for the track-based muon alignment.

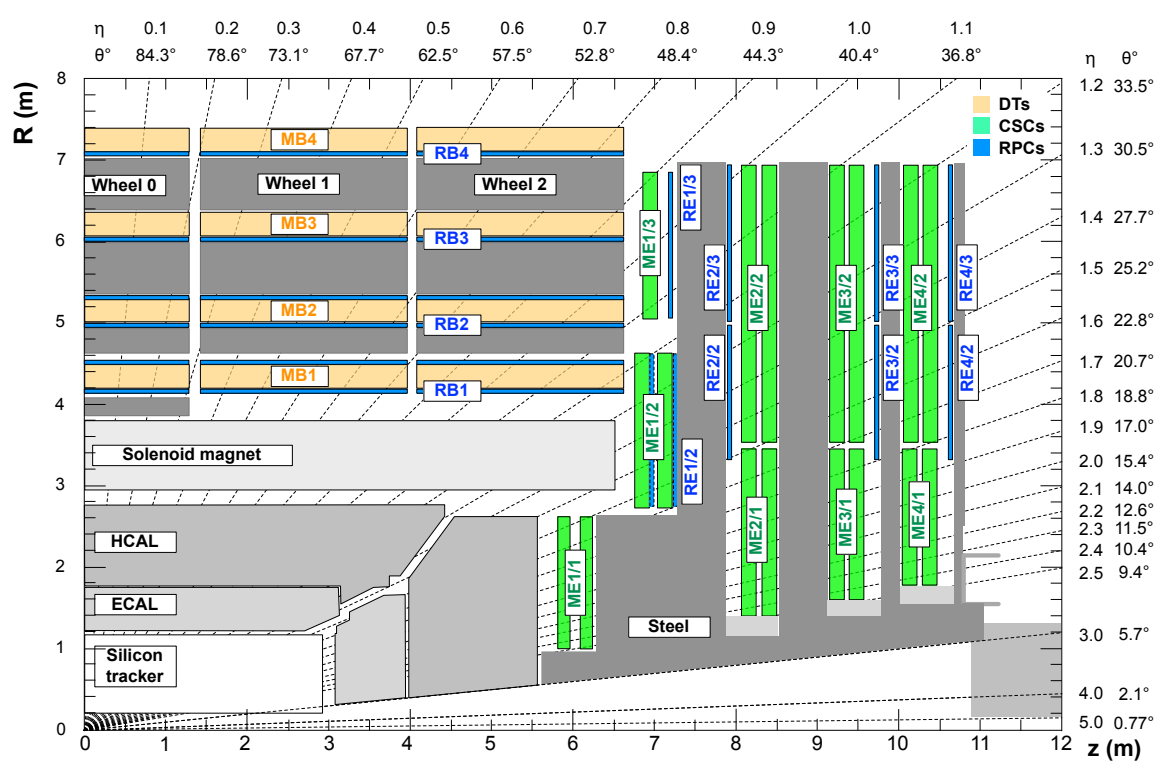

Figure 1: The CMS Muon System.

A more detailed description of the CMS detector, together with a definition of the coordinate system used and the relevant kinematic variables, can be found in Ref. [1].

\section{Track-Based Muon Alignment}

The CMS track-based muon alignment (TBMA) [2] uses the CMS inner tracker tracks and propagates them to the muon system. The predicted hit positions in the muon chambers based on 
Table 1: Muon selection.

\begin{tabular}{ll} 
Muon selection & \\
\hline Transverse momentum $\left(p_{\mathrm{T}}\right)$ & $30 \mathrm{GeV}<p_{\mathrm{T}}<200 \mathrm{GeV}$ \\
Pseudorapidity $(\eta)$ & $|\eta|<2.4$ \\
& \\
Muon track selection & 10 \\
\hline Minimum number of inner tracker hits & 2 \\
Minimum number of matched muon stations & 10 \\
Maximum $\chi^{2}$ (normalized by number of DOF) & 10 \\
Maximum track impact parameters $\left(D_{x y}\right)$ & 0.2
\end{tabular}

the tracker tracks are compared with the reconstructed muon hit positions. The residual between the positions are the input data for the muon alignment. The TBMA minimizes the residuals with a multidimensional objective function effectively for the chamber misalignment degrees of freedom (DOF) $\mathbf{p}=\left(\delta x, \delta y, \delta z, \delta \phi_{x}, \delta \phi_{y}, \delta \phi_{z}\right)$ in local coordinates of the chamber, as shown in Fig 2. The relation between the residuals under investigation and the alignment variables are tested by the alignment ansatz function, please see [2]. The equations 1 and 2 show the cases for DT and CSC, respectively. In these equations the residuals under investigation are on the left side (the red boxes) and the projection matrix $\boldsymbol{M}$ acts on the vector of alignment variables $\mathbf{p}$ (the blue boxes). Eq. 1 shows the DT residuals, a projection matrix $\boldsymbol{M}$, and the misalignment parameters $\mathbf{p}$. The DT stations 1, 2 and 3 provide 2-dimensional hit positions, while the station 4 has only a 1-dimensional hit position, so the residual $\Delta y$ and alignment variables $\delta y$ are not available. The CSC chambers have radial strips, so the residual $\Delta x$ is replaced with the arc length residual $\Delta(R \phi)$. Eq 2 shows the CSC residuals, a projection matrix, and the the alignment variables. The alignment variables contribute to the residuals and their coefficients are represented in a projection matrix $\boldsymbol{M}$. In order to minimize the residuals, the MINUIT [3] algorithm calculates the optimal alignment variables for every chamber. Fig. 3 shows how the CSC residuals are changed after the performed minimization. In order to perform TBMA, a high purity set of muon candidates is collected using the track and muon selection criteria summarized in Table 1.

$$
\left(\begin{array}{c}
\hline \Delta x \\
\hline \Delta y \\
\Delta \frac{d x}{d z} \\
\Delta \frac{d y}{d z}
\end{array}\right)=\left(\begin{array}{cccccc}
1 & 0 & -\frac{d x}{d z} & -y \frac{d x}{d z} & x \frac{d x}{d z} & -y \\
0 & 1 & -\frac{d y}{d z} & -y \frac{d y}{d z} & x \frac{d y}{d z} & x \\
0 & 0 & 0 & -\frac{d x}{d z} \frac{d y}{d z} & 1+\left(\frac{d x}{d z}\right)^{2} & -\frac{d y}{d z} \\
0 & 0 & 0 & -1-\left(\frac{d y}{d z}\right)^{2} & \frac{d x}{d z} \frac{d y}{d z} & \frac{d x}{d z}
\end{array}\right)\left(\begin{array}{c}
\delta x \\
\hline \delta y \\
\hline \delta z \\
\delta \delta \phi_{x} \\
\hline \delta \phi_{y} \\
\hline \delta \phi_{z}
\end{array}\right)
$$




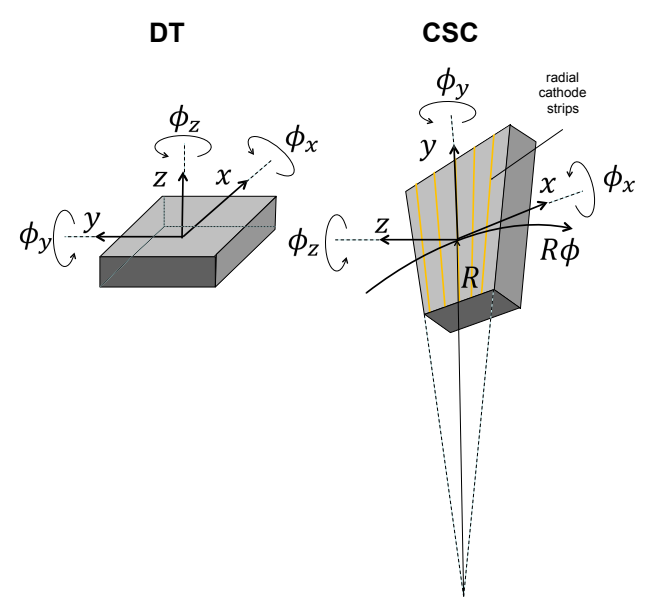

Figure 2: DT and CSC chamber geometries are shown with the possible misalignment degrees of freedom considered.

$$
\left(\begin{array}{c}
\frac{\Delta(R \phi)}{\Delta y} \\
\frac{\Delta \frac{d(R \phi)}{d z}}{\Delta \frac{d y}{d z}}
\end{array}\right)=\left(\begin{array}{cccccc}
1 & \left.-\frac{x}{R}+3\left(\frac{x}{R}\right)^{3}\right] & -\frac{d x}{d z} & -y \frac{d x}{d z} & x \frac{d x}{d z} & -y \\
0 & 1 & -\frac{d y}{d z} & -y \frac{d y}{d z} & x \frac{d y}{d z} & x \\
0 & -\frac{1}{2 R} \frac{d x}{d z} & 0 & {\left[\frac{x}{R}-\frac{d x}{d z} \frac{d y}{d z}\right]} & 1+\left(\frac{d x}{d z}\right)^{2} & -\frac{d y}{d z} \\
0 & 0 & 0 & -1-\left(\frac{d y}{d z}\right)^{2} & \frac{d x}{d z} \frac{d y}{d z} & \frac{d x}{d z}
\end{array}\right)\left(\begin{array}{c}
\frac{\delta x}{\delta \delta y} \\
\delta z \\
\delta \phi_{x} \\
\delta \phi_{y} \\
\delta \phi_{z}
\end{array}\right)
$$
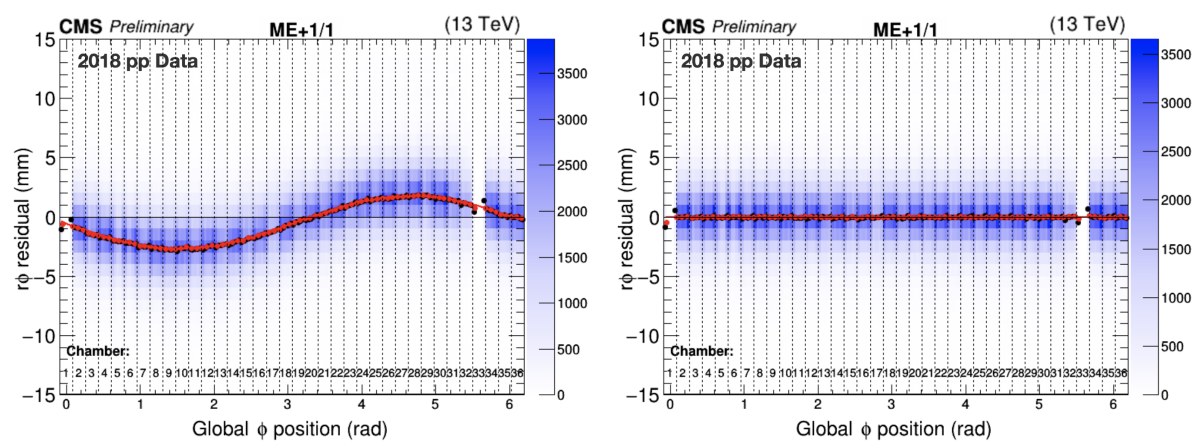

Figure 3: Residual on $r \phi$ as a function of global $\phi$ for the first ring on the first station of CSC chambers in the positive endcap $(\mathrm{ME}+1 / 1)$. On the left, the residual distribution has a sinusoidal trend due to the misalignment of the CSC station. On the right, the residual distribution is centered around zero after the initial geometry has been corrected. The residual means (red), medians (black), and distributions (blue heat map) are shown before alignment (left) and after alignment (right).

The CMS TBMA has been performed on the DT and CSC systems at the chamber level. The CMS muon system alignment method is proven to be efficient, robust, and stable in Run 1 and Run 
2. Furthermore, sources of possible systematic uncertainties have been investigated and various improvements to reduce their effect have been developed and further improvements are in progress. The muon system alignment results (Fig. 4) with the CMS detector simulation shows the CMS muon system alignment accuracy is comparable with the reconstructed hit spatial resolution [4]. Additionally, the results show the alignment accuracy depends on statistics and detector position and type. The TBMA requires at least $2 \mathrm{fb}^{-1}$ or higher integrated luminosity.
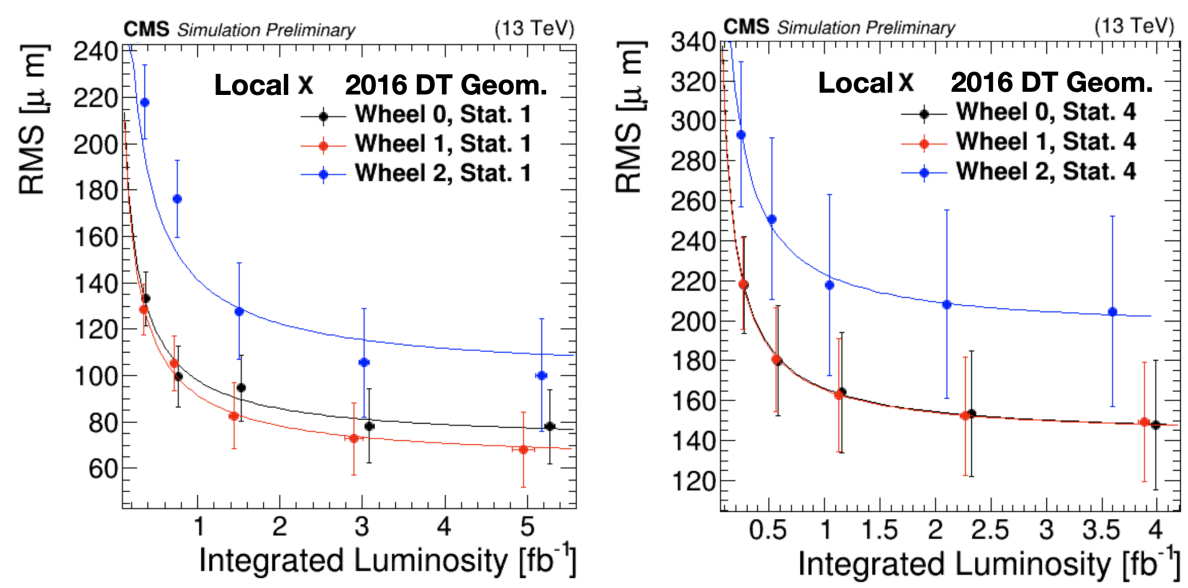

Figure 4: RMS values of misalignment amount in local $\mathrm{X}$ in the CMS track-based muon alignment (TBMA) procedure on DT chambers as a function of the integrated luminosity of pp collisions. This indicates $2 \mathrm{fb}^{-1}$ or larger integrated luminosity is a requirement for TBMA. Each error bar includes systematics uncertainties, such as chamber-to-chamber deviation. The alignment accuracy depends on the detector location (wheels and stations for DT) and six alignment variables ( $\delta x, \delta y, \delta z, \delta \phi_{x}, \delta \phi_{y}$, and $\delta \phi_{\mathrm{z}}$ for DT). Here $\delta x$ is the most sensitive in the $p_{\mathrm{T}}$ resolution, and the best alignment accuracies are obtained from chambers in the central region (Wheel 0 and \pm 1 ).

\section{Run 2 Performance}

To evaluate the alignment results, a physics validation is performed with dimuon events. The invariant mass of dimuon pairs is computed by reconstructing one muon using only muon system information (standalone muon track, STA) and the other using both the tracker and the muon system information (global muon track, GLB). Since one leg of the dimuon is STA, these dimuon mass distributions are sensitive to the muon alignment results. Fig. 5 shows the dimuon mass and mass width distributions by the pseudorapidity of the STA track $\left(\eta_{\mu}^{\mathrm{STA}}\right)$. In order to validate the muon alignment performance, the invariant dimuon mass distribution is not corrected by any scale factors. The Run 2 legacy alignment which is intended to be the final alignment used for all future physics analysis has been improved by using updated tracker legacy geometries, an increased alignment frequency ( 1 alignment/year $\rightarrow 3$ alignments/year), and higher integrated luminosities. 

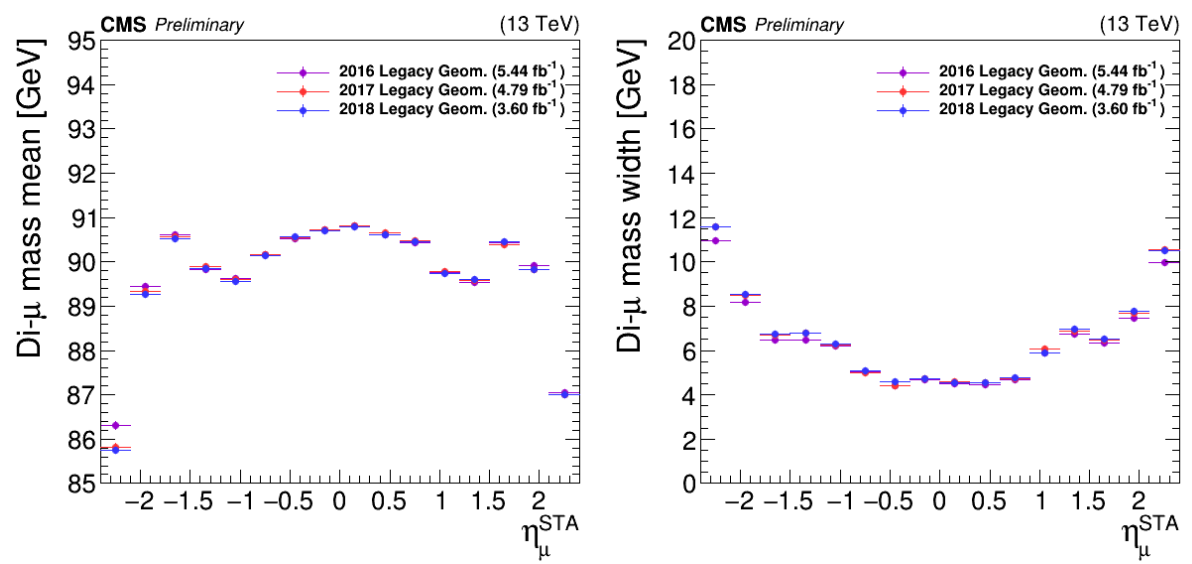

Figure 5: Validation of Run 2 legacy muon alignment using dimuon (GLB + STA) mass distributions for $\mathrm{Z} \rightarrow \mu^{+} \mu^{-}$event candidates. Left: Dimuon mass (mean and width values from Gaussian fit) as a function of the standalone muon track pseudorapidity $\left(\eta_{\mu}^{\mathrm{STA}}\right)$. Right: Dimuon mass width as a function of $\eta_{\mu}^{\mathrm{STA}}$. High $\eta$ (endcap) region is unstable and sensitive to operating conditions. Since one dimuon leg is STA, there is scale bias (a $1 \%$ scale bias in barrel and a up to $5 \%$ bias in the endcap [4]).

\section{Summary}

The performance of the track-based CMS muon system alignment has been reported using muons in proton-proton collisions at the LHC. High purity events and a set of high-quality tracks were used to align muon chambers by minimizing the residuals calculated from muon hits and propagation of inner tracker tracks. The performance of the track-based muon alignment in Run 2 was robust and stable. The physics-based performance of alignments has been demonstrated with special reconstruction of the dimuon mass and width using data. The muon alignment supported excellent muon reconstruction in CMS and contributed to many physics analyses with muons.

\section{References}

[1] CMS Collaboration, The CMS experiment at the CERN LHC, JINST 3 (2008) S08004.

[2] CMS Collaboration, Alignment of the CMS muon system with cosmic-ray and beam-halo muons, JINST 5 (2010) T03020.

[3] F. James and M. Roos, Minuit - a system for function minimization and analysis of the parameter errors and correlations, Computer Physics Communications 10 (1975) 343.

[4] CMS Collaboration, Performance of the CMS muon detector and muon reconstruction with proton-proton collisions at $\sqrt{s}=13 \mathrm{TeV}$, JINST 13 (2018) P06015. 